\title{
Maternal diet, LCPUFA status and prematurity in Indian mothers: A cross-sectional study
}

\author{
Prachi S. Ranade ${ }^{1}$, Shobha S. Rao ${ }^{1,2}$ \\ ${ }^{1}$ Biometry and Nutrition unit, Animal Science Division, Agharkar Research Institute, G.G. \\ Agarkar Road, Pune-411004, India
}

${ }^{2}$ Corresponding author: Shobha S. Rao, PhD, Biometry and Nutrition unit, Agharkar Research Institute, Pune 411004, India

Submission date: August 21, 2012, Acceptance date: November 15, 2012; Publication date: November 16, 2012

\begin{abstract}
Background: Recently, certain long chain polyunsaturated fatty acids (LCPUFA) have been shown to exert functional benefits with regards to gestational length. The present study examined the association of maternal LCPUFA intake, specifically Docosahexaenoic acid (DHA) and Arachidonic acid (ARA), and plasma status at delivery with duration of gestation and risk of premature delivery in Indian mothers.
\end{abstract}

Methods: In a cross-sectional nested case-control study, 191 mother-baby pairs [164 Full term (FT) and 27 Preterm (PT)] were examined for differences in maternal habitual diet pattern and plasma fatty acid composition in relation to the length of gestation.

Results: The frequency of intake of $n-3$ fatty acid rich varieties of fish was higher $(p<0.05)$ in FT mothers compared to PT mothers. Maternal plasma fatty acid concentration of n-3 Alpha Linolenic acid (ALA), Eicosapentaenoic acid (EPA), DHA and total n-3 fatty acids at delivery was significantly associated with intake of vegetarian ALA sources such as millets, dark whole pulses, dry fruits like walnuts, and green leafy vegetables. Among age, parity, and neonatal sex matched case-control pairs, PT mothers had significantly $(\mathrm{p}<0.01)$ higher levels of $\mathrm{n}-6$ ARA, but lower $(\mathrm{p}<0.01)$ levels of n-3 DHA and total n-3 fatty acids compared to FT mothers irrespective of socioeconomic group. In fact, mothers with plasma DHA levels below median $(<3.0 \%)$ had ten times higher risk (OR-10.47; 95\% CI: 3.03-36.48) of delivering prematurely compared to those who had plasma DHA levels above median.

Conclusion: Results underscore the importance of consuming varied sources of ALA and DHA for their role as functional lipids in determining gestational length.

Key words: LCPUFA, maternal diet, omega 3, DHA, preterm birth, relative risk 


\section{BACKGROUND}

The incidence of preterm delivery is $10-11 \%$ in developed countries like USA [1] and is reported to vary considerably (12-27\%) in India [2-4]. Prematurity is a leading cause of low birth weight and perinatal morbidity and mortality, in both developed as well as developing countries [5]. A wide spectrum of causes and demographic factors like parity [6], extremes of maternal age [7], poor maternal height, weight, BMI, and pregnancy weight gain [8], anemia during pregnancy [9], vaginal or urinary infection [10], and macro or micronutrient deficiencies [11] have been implicated in the etiology of prematurity.

Recent evidence from human and animal studies indicates that LCPUFA, of both n-3 and n6 series and their eicosanoid metabolites, work as functional lipids and play important modifiable roles in gestational duration. For example, observational and supplementation studies in the Danish population presented promising association between fish and fish oil consumption and increase in gestational length in normal [12-15] as well as high risk pregnancies [16]. In pregnant sheep models, intravenous infusion of fish oil during late gestation prevented, delayed, and in some cases reversed drug-induced premature delivery [17]. Conversely, RCTs of n-3 LCPUFA supplementation in Norwegian [18] and British [19] pregnant women have found no effect on length of gestation. The inconsistent effects on pregnancy outcome noted in case of maternal supplementation studies possibly point to the fact that, short term interventions or pharmacological doses may have limitations in conferring the desired effects as compared to those achieved due to habitual yet minimal consumption from dietary sources.

Most of the reported human studies linking n-3 fatty acid intake with better birth outcome have been performed among populations with very high seafood intake [12-14]. Therefore their results may not be transferable to other countries like India, where there is predominance of nonfish eating communities. Hence, investigating the role of precursors of functional lipids, like essential fatty acids (EFA) and their long chain derivatives, in reducing risk of adverse pregnancy outcome becomes important. We carried out a cross-sectional study with the aim to assess the association between maternal EFA and LCPUFA intake and premature delivery status.

\section{METHODS:}

Study design: The study was performed in collaboration with the Obstetrics and Gynecology department of Deenanath Mangeshkar Hospital, Pune, India. Clearance was obtained from the Institutional Research Ethics Committee of the hospital for the cross-sectional, nested casecontrol study. Sample size was calculated from a community based longitudinal study of pregnant rural Indian women wherein the rate of preterm delivery was approximately $11 \%$ [20] using power of $80 \%$ and $\mathrm{p}=0.05$. Preterm birth was defined as gestational age $<37$ weeks by Last Menstrual Period (LMP), based on clinical and sonographic evaluation. Deliveries prior to completion of 37 gestational weeks were considered to be premature deliveries.

Subjects: Pregnant women attending the Ante Natal Care (ANC) clinic during pregnancy and who registered for delivery in the hospital were enrolled for the study. Only women who had no prior history of preterm labor or delivery were included for the study, whereas women with established history of medical risk factors like pre-eclampsia, gestational diabetes, HIV or 
multiple gestations were excluded. For preterm cases, we excluded medically indicated preterm birth and included only spontaneous and or induced preterm deliveries due to preterm premature rupture of membrane (PPROM). All participants were briefed about the study and informed, signed consent was obtained according to the Ethical Guidelines for Biomedical Research on human participants [21]

Maternal data collection: Baseline data included personal information like date of birth, age of marriage, family type, family size, socio-economic information pertaining to family income, education, occupation, and assets, and was collected with the help of a standardized pre-test pro forma. Obstetric history, like parity and LMP, were also recorded. Gestational age was calculated from LMP and confirmed by clinical evaluation and sonography reports. Early pregnancy weight and height as well as serial measurements of weight until delivery were recorded from the subjects' ANC follow-up notes.

Dietary pattern: The participants' typical dietary intake was recorded by carefully administering a specifically designed and validated food frequency questionnaire (FFQ) [20]. The frequency of intake of nearly 130 food items from 10 different food groups, ranging from "never" to "twice/thrice a day" was obtained for the reference period of one month prior to the interview. The 10 food categories were: cereals/millets and products, pulses and legumes, poultry/meat/seafood, dairy products, other/green leafy vegetables, fruits, snacks (sweet \& spicy), oilseeds, dry fruits and fats, special foods for pregnancy, and omega 3 supplements. These food categories were mutually exclusive and each category contained commonly consumed EFA and LCPUFA rich foods. Frequency of consumption of individual food items was converted into scores, and then scores for each food category was obtained by adding the scores of various foods in it. Thus in our study, the FFQ allowed us to compare the groups of women consuming relatively low and high frequency of specific food groups and foods rich in EFA and LCPUFA.

Blood collection at delivery: $10 \mathrm{cc}$ blood samples were collected from all enrolled mothers who were admitted for delivery at the hospital during the routine sample collection for crossmatching. Blood was collected in evacuated tubes containing EDTA and immediately refrigerated. Blood samples were processed within $24 \mathrm{~h}$ of collection, and plasma aliquots were labeled and stored at $-80^{\circ} \mathrm{C}$ until analysis.

Fatty acid analysis of maternal plasma: Maternal plasma total lipids were extracted by the Folch method [22]. Fatty acid methyl esters were prepared by AOAC method [23] using $0.5 \mathrm{~N}$ $\mathrm{H}_{2} \mathrm{SO}_{4} /$ methanol reagent. Lipid samples were mixed with $1 \mathrm{ml}$ hexane in $16 \mathrm{ml}$ glass tubes with Teflon-lined caps. $\mathrm{H}_{2} \mathrm{SO}_{4} / \mathrm{MeOH}$ reagent $(1 \mathrm{ml})$ was added, and the mixture was heated at 90 $110^{\circ} \mathrm{C}$ in a water bath for 1 hour, cooled to room temperature and methyl esters extracted in the hexane phase after addition of $1 \mathrm{ml} \mathrm{H} 2 \mathrm{O}$. Samples were allowed to stand for 20-30 min, and then the upper hexane layer was removed and concentrated under nitrogen. The total lipid fatty acid composition was analyzed by Gas Chromatography using Shimadzu model GC-17A 
Version 3 (Kyoto Japan) fitted with Omegawax 25 capillary column, $30 \mathrm{~m}$ x $0.32 \mathrm{~mm}$ i.d, $0.25 \mathrm{ft}$. Temperature program was as follows: initial temperature $100^{\circ} \mathrm{C} \mathrm{(5} \mathrm{minute} \mathrm{hold),} 100$ to $220^{\circ}$ at the rate of $10^{\circ} \mathrm{C} \mathrm{min}^{-1}$, and hold at $220^{\circ}$ for 12 minutes. Injector temperature was $200^{\circ} \mathrm{C}$, and the detector temperature was $230^{\circ} \mathrm{C}$. Detector was FID. Carrier gas was helium with flow rate of $1.8 \mathrm{ml} / \mathrm{min}$ and split ratio of 33:1. Fatty acid concentrations were calculated from their relative peak areas as compared to internal standard. Retention times and peak areas were automatically computed by GCMS. Individual fatty acids were identified by comparing the retention time of peak with that of standard fatty acid methyl esters (37 component FAME mixture, Sigma, USA). Fatty acids were quantified as percent total fatty acids in the sample lipids.

Statistical analysis: The baseline maternal information on occupation, education of self and spouse and possession of economic assets such as owning a house, car, telephone etc., was obtained on 3-point scale each so as to discriminate between income classes, using a pretested validated pro forma [24]. Socioeconomic (SE) information of the participating mothers was coded by assigning weighted scores to different indicators to develop a SE score and define the socioeconomic status (high - HSE or low - LSE) of the mother. SE score was used as a categorical variable to compare mothers from HSE and LSE class. Food frequency data was quantified into frequency scores for each food group, as described above. The FFQ scores were standard numerical values which were used to compute the tertile and quartile distribution of mothers according to their consumption pattern. Continuous variables: namely maternal age, gestational length, as well as maternal plasma fatty acid profile, were expressed as mean \pm SD or SEM, unless specified otherwise. Categorical variables were defined using standard cut-offs for preterm birth for comparison between groups. Variables with abnormal distribution were normalized with logarithmic or square root transformation. Differences between means of FT HSE, FT LSE and PT groups were tested by ANOVA. Students' t-test was used for comparison between FT and PT neonates and for mean values of gestation by percentiles of maternal fatty acid profile. Significance was defined at 0.05 level of confidence. Gestational length was adjusted for maternal confounding factors like age, BMI, parity by regression analysis prior to computing Odds ratio for risk of preterm delivery. All statistical analyses were performed by using SPSS/PC+, Version 11.0 (SPSS Chicago, IL).

\section{RESULTS:}

Maternal and neonatal characteristics: There was no significant difference between mothers from FT and PT group for age at delivery, pre-pregnancy BMI, weight gain up to $3^{\text {rd }}$ trimester or type of labor. There was no difference in male: female ratio of FT and PT neonates (Table 1).

Maternal food habits and dietary intake of LCPUFA: We examined differences in maternal diet patterns in FT and PT group. It can be seen that the majority of the mothers were Lacto-ovo or non-vegetarian consumers with a similar trend noted in FT as well as PT groups (Table 2). Mothers from the PT group consumed meat, poultry or its products more frequently, and fish less frequently, than mothers from FT group. 
Table 1 Maternal and neonatal characteristics in term and preterm cases

\begin{tabular}{lccc}
\hline Variables & $\begin{array}{c}\text { Term } \\
(\mathbf{n = 1 6 4})\end{array}$ & $\begin{array}{c}\text { Preterm } \\
(\mathbf{n = 2 7})\end{array}$ & $\begin{array}{c}\text { P- } \\
\text { value }\end{array}$ \\
\hline Maternal characteristics & & & \\
& & & $\mathrm{ns}$ \\
Age (yr) & $26.9 \pm 3.7$ & $25.4 \pm 5.7$ & $\mathrm{~ns}$ \\
Pre-pregnancy BMI $\left(\mathrm{kg} / \mathrm{m}^{2}\right)$ & $23.1 \pm 3.5$ & $23.0 \pm 3.2$ & $\mathrm{~ns}$ \\
Weight gain up to 30 weeks of pregnancy (kg) & $9.1 \pm 2.8$ & $8.3 \pm 3.1$ & $\mathrm{~ns}$ \\
Type of labor/delivery & & & $\mathrm{ns}$ \\
$\quad$ Spontaneous & $115(70.1)^{*}$ & $21(77.8)$ & \\
$\quad$ Induced & $49(29.9)$ & $06(22.2)$ & $\mathrm{ns}$ \\
\hline Neonatal characteristics & & & $\mathrm{ns}$ \\
Gestational age at birth (days) & $276.7 \pm 7.7$ & $241.3 \pm 17.9$ & \\
Males & $90(54.9)$ & $13(48.1)$ & \\
Females & $74(45.1)$ & $14(51.9)$ & \\
$\quad$ ns, non-significant & & &
\end{tabular}

Table 2 Maternal food habits in term and preterm cases

\begin{tabular}{llll}
\hline Food habit & Term $(\mathbf{n = 1 6 4})$ & Preterm $(\mathbf{n = 2 7})$ & P- value \\
\hline Vegetarian & $70(42.7)^{*}$ & $9(33.3)$ & $\mathrm{ns}$ \\
Lacto-ovo-vegetarian & $16(9.8)$ & $2(7.4)$ & $\mathrm{ns}$ \\
Non-vegetarian & $78(47.6)$ & $16(59.3)$ & $\mathrm{ns}$ \\
\hline Frequency of consumption of non-vegetarian foods & & \\
Meat & $142(86.6)$ & $19(70.4)$ & 0.01 \\
Never & $17(10.4)$ & $7(25.9)$ & 0.01 \\
<W 1 & $5(3.0)$ & $1(3.7)$ & $\mathrm{ns}$ \\
W 1 & $0(0.0)$ & $0(0.0)$ & $\mathrm{ns}$ \\
>W 1 & & $11(40.7)$ & $\mathrm{ns}$ \\
Poultry & $92(56.1)$ & $15(55.6)$ & 0.02 \\
Never & $58(35.4)$ & $1(3.7)$ & $\mathrm{ns}$ \\
<W 1 & $14(8.5)$ & $0(0.0)$ & $\mathrm{ns}$ \\
W 1 & $0(0.0)$ & & \\
$>$ W 1 & & $25(92.6)$ & 0.02 \\
Fish & $128(78.0)$ & $1(3.7)$ & 0.01 \\
Never & $31(18.9)$ & $1(3.7)$ & $\mathrm{ns}$ \\
<W 1 & $3(1.8)$ & $0(0.0)$ & $\mathrm{ns}$ \\
W 1 & $2(1.2)$ & &
\end{tabular}

ns, non-significant; *Figures in parenthesis indicate percentage 
It is known that fresh water and salt water fish differ greatly in their fatty acid profile, and certain shellfish like oysters and crustaceans like crabs are also good sources of omega-3 fatty acids [25]. Our dietary data revealed considerable differences in the type of fish consumption in mothers from the FT and PT group (Figure 1). A significantly greater number of mothers from the PT group (55\%) consumed fresh water fish like crap and catla, compared to mothers from FT group $(20 \%)$. A reverse trend was noted in case of salt water fish, where mothers from FT group were seen to consume salt water varieties like bombay duck and pomfret. Fatty fish like king mackerel, Indian salmon and sardine which are reported to have the greatest quantities of omega3 fatty acids [25] were consumed mainly by mothers in the FT group (30\%). Similarly, crab, shrimp, oysters and clams which belong to the filter feeding 'molluscs' family and derive their fatty acids directly from the phytoplankton; were consumed more by mothers in FT group.

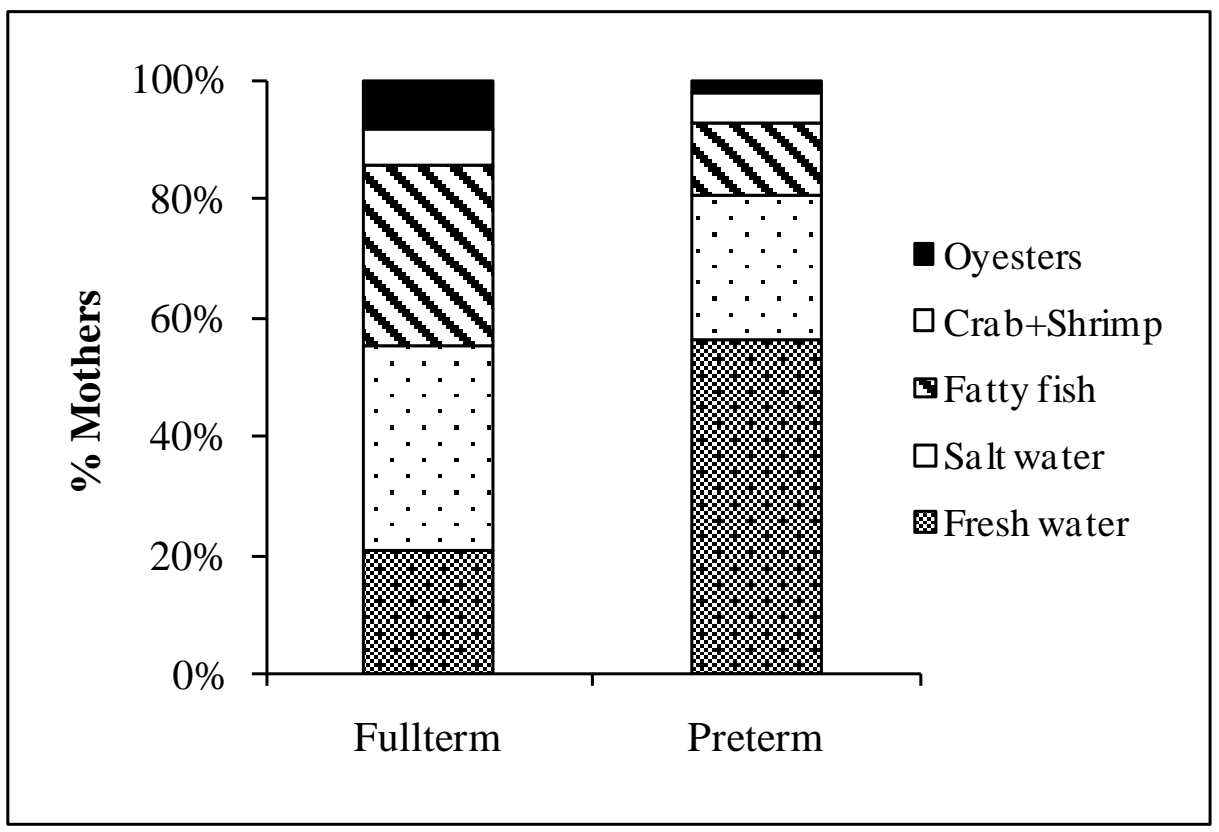

Figure 1 Type of fish consumed by mothers delivering at term and preterm

Dietary information pertaining to fish consumption collected from 167 Full Term and 27 Preterm delivering mothers using customized Food frequency questionnaire (FFQ).

Maternal plasma fatty acid status: The fatty acid status in women is known to be affected by their age, parity [26] and socioeconomic factors [27]. Therefore, we matched mothers in FT and PT group for these confounders, and comparisons were made only between matched cases and controls. Plasma fatty acid profiles of FT (HSE) and FT (LSE) mothers were comparable (Table 3). Further, there was no difference in the levels of saturated or monounsaturated fatty acids amongst FT (HSE), FT (LSE) and PT group mothers. The plasma content of essential fatty acids, namely LA and ALA, was also similar in these three groups. However, the LCPUFA profile was considerably different between the FT and PT mothers. PT mothers had significantly $(\mathrm{p}<0.01)$ higher levels of n-6 ARA, but lower $(p<0.01)$ levels of $n-3$ DHA, and total $n-3$ fatty acids compared to both FT (HSE) and FT (LSE) group mothers. The total PUFA content of plasma was higher in case of PT mothers, as was the $n 6 / n 3$ ratio. 
Table 3 Maternal plasma fatty acid composition (\%) in full term and preterm cases

\begin{tabular}{llll}
\hline Selected fatty acids & $\begin{array}{c}\text { FT HSE } \\
(\mathbf{n = 2 3})\end{array}$ & $\begin{array}{c}\text { FT LSE }^{\$} \\
(\mathbf{n = 2 1})\end{array}$ & $\begin{array}{c}\text { PT }^{\$} \\
(\mathbf{n}=\mathbf{2 7})\end{array}$ \\
\hline 12:0 Lauric acid & $0.70 \pm 0.3$ & $0.67 \pm 0.3$ & $0.89 \pm 0.6$ \\
14:0 Myristic acid & $1.10 \pm 0.4$ & $1.00 \pm 0.2$ & $1.1 \pm 0.5$ \\
16:0 Palmitic acid & $38.3 \pm 2.9$ & $36.7 \pm 3.1$ & $35.8 \pm 3.7$ \\
16:1(n-9) Palmitoleic acid & $1.0 \pm 0.5$ & $1.1 \pm 0.6$ & $1.2 \pm 0.5$ \\
18:0 Stearic acid & $8.8 \pm 1.6$ & $10.1 \pm 1.1$ & $10.2 \pm 1.5$ \\
18:1(n-9) Oleic acid & $15.4 \pm 1.7$ & $14.3 \pm 1.5$ & $13.2 \pm 2.0$ \\
18:2 (n-6) LA & $22.7 \pm 2.2$ & $24.3 \pm 3.7$ & $23.8 \pm 3.1$ \\
18:3 $(n-3)$ ALA & $2.0 \pm 0.6$ & $1.9 \pm 0.1$ & $2.2 \pm 0.5$ \\
20:4 $(n-6)$ ARA & $5.1 \pm 0.8$ & $5.5 \pm 0.3$ & $8.0 \pm 1.5^{* *}$ \\
20:5 $(n-3)$ EPA & $1.2 \pm 0.2$ & $1.1 \pm 0.2$ & $1.2 \pm 0.4$ \\
22:6 $(n-3)$ DHA & $3.0 \pm 0.6$ & $2.8 \pm 0.6$ & $1.9 \pm 0.6^{* *}$ \\
Total SFA & $49.0 \pm 3.0$ & $48.5 \pm 3.6$ & $48.0 \pm 3.2$ \\
Total MUFA & $16.4 \pm 2.7$ & $15.5 \pm 2.0$ & $14.4 \pm 2.2$ \\
Total n-6 fatty acids & $27.8 \pm 3.7$ & $29.8 \pm 3.0$ & $31.9 \pm 3.2$ \\
Total n-3 fatty acids & $6.3 \pm 1.2$ & $5.9 \pm 0.8$ & $5.3 \pm 1.2^{* *}$ \\
Total PUFA & $34.2 \pm 1.7$ & $5.8 \pm 3.1$ & $37.2 \pm 3.4^{* *}$ \\
$\quad$ n6/n3 ratio & & $6.3 \pm 1.5^{* *}$ \\
\hline
\end{tabular}

$\$$ matched for mothers age, parity and sex of child

** $\mathrm{p}<0.01$ significantly different from FT HSE and FT LSE mothers

SFA; saturated fatty acids, MUFA; monounsaturated fatty acids

Maternal plasma fatty acid profile in relation to dietary EFA and LCPUFA intake: Based on the data of fatty acids present in commonly consumed Indian vegetarian foods [28], a total of five food categories which are reported to be good source of n-3 fatty acids were considered for the analysis viz., cereal and millets, whole pulses, oilseeds and dry fruits, green leafy vegetables and seafood.

Maternal plasma fatty acid levels according to frequency of consumption of selected foods mentioned above are presented in Table 4. 
Table 4 Maternal plasma fatty acid levels according to frequency of consumption of selected foods

\begin{tabular}{llll}
\hline Food group and food & Frequency of consumption & $\begin{array}{l}\text { Plasma fatty } \\
\text { acid level }\end{array}$ & $\begin{array}{l}\text { P- } \\
\text { value }\end{array}$ \\
\hline \multirow{2}{*}{$\begin{array}{l}\text { Rice and or products } \\
\text { Wheat and or products }\end{array}$} & $>2 /$ day Vs. $<2 / \mathrm{d}$ & $\uparrow$ LA & 0.091 \\
& $>2 /$ day Vs. $<2 / \mathrm{d}$ & $\downarrow$ LA & $0.018^{*}$ \\
& Highest tertile Vs. Lowest tertile & $\uparrow$ ALA & $0.049^{*}$ \\
& Highest tertile Vs. Lowest tertile & $\uparrow$ DHA & $0.002^{*}$ \\
Millets and or products & Daily (5-6 times/ week) Vs. Once/ week & $\uparrow$ ALA & $0.023^{*}$ \\
& & $\uparrow$ EPA & $0.002^{*}$ \\
& & $\uparrow$ DHA & $0.000^{*}$ \\
Whole pulses and legumes & $>$ Once/week Vs. $<$ once/week & $\downarrow$ ARA & 0.090 \\
Green leafy vegetables & Daily (5-6 times/ week) Vs. once/ week & $\uparrow$ ALA & 0.089 \\
Seafood & Never Vs. once/ week & $\uparrow$ DHA & $0.049^{*}$
\end{tabular}

Comparison of means using Students' T-test

* Significantly different at $\mathrm{p}<0.05$

$\uparrow$ Increase in mean plasma levels; $\downarrow$ decrease in mean plasma levels

Mothers with higher consumption (> twice a day) of rice or its products had marginally higher $(\mathrm{p}=0.091)$ plasma LA compared to mothers who consumed it once a day $(23.6 \%$ vs. $23.0 \%)$. Conversely, higher consumption of wheat products was associated with significantly ( $p$ $=0.018$ ) lower plasma LA concentrations. Further, mothers in highest tertile of wheat consumption also had better plasma n-3 status than those in lowest tertile, as reflected from significantly greater concentration of ALA (2.10\% vs. $1.91 \%, \mathrm{p}=0.049)$ and DHA (3.0\% vs. $2.6 \%, \mathrm{p}=0.002)$. We observed that mothers who had millet based foods on a daily basis $(5 / 6$ times a week) had significantly better plasma n-3 profile than mother who consumed millets once in a week. They had higher ALA (2.1\% vs. 1.9\%, p =0.023), EPA (1.3\% vs. 1.1\%, p = $0.002)$, DHA (3.15\% vs. $2.53 \%, \mathrm{p}=0.000)$ and lower ARA $(5.44 \%$ vs. $5.80 \%, \mathrm{p}=0.09)$. With regard to whole pulses and legumes, higher consumption showed marginally better $(\mathrm{p}=0.089)$ plasma DHA levels than those with lower (< once a week) consumption. Similarly, daily consumption of either oilseed, spices, or dry fruit, was marginally associated with better plasma DHA status compared to weekly consumption $(2.93 \%$ vs. $2.76 \%, \mathrm{p}=0.089)$. In our study, $85 \%$ of participating mothers consumed some type of green leafy vegetables (GLV) at least once a week. Thus mothers who ate GLVs on a daily basis (five times a week) had significantly ( $\mathrm{p}=$ 0.049) higher plasma ALA (2.05\% vs. $1.95 \%)$ than mothers who had GLV on a weekly basis. For the last food group i.e. fish and seafood we compared plasma fatty acid profile of vegetarian 
mothers who never consumed these foods with those who consumed it on a weekly basis. Although we found no difference in plasma levels of LA, ALA, ARA and EPA in the two groups, plasma DHA levels were significantly higher in vegetarian mothers $(2.90 \%$ vs. $2.69 \%, \mathrm{p}$ $=0.038$ ).

Gestational length as a function of maternal EFA and LCPUFA status: In order to assess the risk levels of maternal plasma and EFA and LCPUFA with respect to gestational length, we compared the mothers below the $10^{\text {th }}$ and above the $90^{\text {th }}$ percentile of plasma fatty acid levels (Table 5).

Table 5 Mean values of gestation by percentiles $\left(<10^{\text {th }}\right.$ and $\left.>90^{\text {th }}\right)$ of maternal fatty acid profile

\begin{tabular}{|c|c|c|}
\hline \multirow[b]{2}{*}{ Selected fatty acids } & \multicolumn{2}{|c|}{ Gestation (days) \# } \\
\hline & Mean & $\mathrm{P}$ value \\
\hline \multicolumn{3}{|l|}{$18: 2(n-6)$} \\
\hline$<10$ th $(19)^{*}$ & 262.3 & ns \\
\hline$>=90$ th $(17)$ & 262.6 & \\
\hline \multicolumn{3}{|l|}{$18: 3(n-3)$} \\
\hline$<10$ th $(18)$ & 265.1 & ns \\
\hline$>=90$ th $(19)$ & 264.5 & \\
\hline \multicolumn{3}{|l|}{$20: 4(n-6)$} \\
\hline$<10$ th (19) & 274.7 & 0.000 \\
\hline$>=90$ th $(19)$ & 244.6 & \\
\hline \multicolumn{3}{|l|}{$22: 6(n-3)$} \\
\hline$<10$ th $(14)$ & 248.5 & 0.000 \\
\hline$>=90$ th $(16)$ & 276.2 & \\
\hline \multicolumn{3}{|l|}{ Total n-6 } \\
\hline$<10$ th $(17)$ & 270.8 & 0.026 \\
\hline$>=90$ th $(20)$ & 256.8 & \\
\hline \multicolumn{3}{|l|}{ Total n-3 } \\
\hline$<10$ th (19) & 259.3 & 0.070 \\
\hline$>=90$ th $(19)$ & 271.3 & \\
\hline
\end{tabular}

\# Adjusted gestational length computed by regression analysis ; adjusted for maternal age, BMI and parity Comparison of means using Students' T-test ns, non significant

*Figures in parenthesis indicate number of cases

Mothers in the latter group for ARA and total n-6 fatty acids had significantly shortened gestation. In contrast, mothers who had $>90^{\text {th }}$ percentile of DHA had 28 days longer gestation than women with DHA $<10^{\text {th }}$ percentile. In fact, mothers with plasma DHA levels below the median level (3\%) had ten times higher risk for preterm delivery (OR-10.47; 95\% CI: 3.0336.48) as compared to mothers with plasma DHA levels greater than or equal to median. 


\section{DISCUSSION:}

Prematurity contributes to considerably more neonatal morbidity and mortality than low birth weight (LBW), and preterm infants fail to reap the benefits of most rapid fetal growth phases associated with this last few weeks of in-utero life, due to their abruptly truncated gestation. Observations that mothers delivering preterm babies have n-3 LCPUFA deficiency [29], and that birth weight and gestational length are associated with maternal DHA consumption and its status, have been reported in western populations [12-16]. Very few studies have assessed maternal LCPUFA intake in context of birth outcome in largely vegetarian populations like India. We observed that maternal plasma fatty acid concentration of n-3 ALA, EPA, DHA and total n-3 fatty acids at delivery was significantly associated with intake of vegetarian ALA sources such as millets, dark whole pulses, dry fruits like walnuts, green leafy vegetables. Further, PT mothers had significantly $(\mathrm{p}<0.01)$ higher levels of n-6 ARA, but lower $(\mathrm{p}<0.01)$ levels of $\mathrm{n}-3$ DHA and total n-3 fatty acids compared to FT mothers irrespective of socioeconomic group.

We observed that maternal age at delivery, pre-pregnancy BMI, weight gain up to $3^{\text {rd }}$ trimester or type of labor did not differ significantly among FT and PT mothers in our study. This observation, however, is not in line with reported literature, where PT mothers were noted to fall in extreme age categories [7], had small stature and slower rate of weight gain [8]. We did not employ purposive sampling method for the study. Hence, the homogenity in the demographic profile of the cases and controls may be attributed to the basic composition of the sample population. In contrast, we noted significant differences in dietary habits between cases (PT) and controls (FT). For example, significantly fewer PT mothers consumed fish, and even their frequency of consumption was less compared to FT mothers. This is in conformation with a study of pregnant women from a south Indian urban cohort where $44 \%$ of study population did not consume fish; and among fish eating women, low consumption of fish during pregnancy was associated with poor birth outcome [30]. Further, the finding that mothers from the PT group consumed considerably less omega-3 rich varieties of fish and shellfish is also supportive to the report on Danish pregnant women wherein low consumption of seafood was a strong predictor of preterm delivery as well as LBW babies [31].

When maternal age, parity, and socioeconomic class were matched, PT mothers had significantly $(\mathrm{p}<0.01)$ higher levels of $n-6$ ARA, but lower $(\mathrm{p}<0.01)$ levels of $\mathrm{n}-3$ DHA and total n-3 fatty acids compared to both FT (HSE) and FT (LSE) group mothers. The total PUFA content of plasma was higher in case of PT mothers, as was the n6/n3 ratio. Reece et al. [29] reported that compared to full term mothers, percent total LA, ARA, EPA and n6/n3 ratio were higher whereas total $\mathrm{n}-3$ fatty acids were lower in preterm cases. PT mothers had $70 \%$ higher ARA levels and 34\% lower DHA levels. Therefore, authors suggested that altered ARA mobilization coupled with omega-3 fatty acid deficiency may play a role in premature labor and parturition. (Araya et al. [32]) also found differences in maternal erythrocyte (RBC) ARA content in term and preterm delivering mothers and hypothesized that high content of maternal RBC ARA and ARA/DHA ratio may be considered as an early signal of preterm delivery. Similar observations are reported in Brazilian [33] and Iraqi mothers [34] who delivered preterm.

Circulating fatty acid levels reflect dietary intake of EFA and LCPUFA over a few days [35]. Millets are known to have high ALA [28]. We observed that mothers consuming them on a 
daily basis had significantly higher ALA, EPA, DHA and lower ARA compared to those who consumed them once a week or less. Similarly, green leafy vegetables (GLVs) have been reported to furnish more ALA than other vegetables and fresh fruits. In fact agathi, colocasia, drumsticks and fenugreek provide 10 times more ALA than other vegetables and fruits and two times more than most of the legumes and pulses [28]. Mothers who consumed GLVs on a daily basis had in fact significantly higher plasma ALA than mothers who consumed them on a weekly basis. Thus even amongst a predominantly vegetarian population, a wide variety of sources of n3 fatty acids in daily diet was associated with better plasma n-3 fatty acid status.

Accumulating evidence suggests that essential fatty acids play important roles in improving pregnancy outcome, and Docosahexaenoic acid derived from alpha linolenic acid is now surfacing as the key modulator of fetal growth. Our study not only brought out that there were significant differences in the n-6 ARA and n-3 DHA levels of PT and FT mothers, but also showed that mothers with plasma DHA levels below median $(<3 \%)$ had ten times higher risk of premature delivery. These observations support the hypothesis that an increase in $n-6$ fatty acid pools and a decrease in n-3 pools may trigger risk for premature delivery and indicate that maternal DHA status could serve as a biomarker of adverse pregnancy outcome.

\section{CONCLUSION:}

To our knowledge, ours is the first study to examine maternal diet and plasma fatty acid status in Indian pregnant women with respect to gestational length and risk of preterm delivery. In this cross-sectional study, an increase in the intake and levels of n-6 fatty acids mainly LA and ARA significantly increased the chance of preterm birth, whereas higher circulating n-3 fatty acids, namely ALA and DHA, in maternal plasma was advantageous with respect to length of gestation. Based on our observations, we propose that measurement of maternal plasma n-3 and DHA levels could be valuable as functional indicators when conducted as a part of ANC checkup. If identified early during pregnancy, it could be possible to ameliorate the deficiency by adopting pragmatic approaches to promote consumption of soybean oil and other sources of ALA like millets and green leafy vegetables especially in malnourished pregnant women from poor socio-economic background.

Abbreviations: Essential fatty acids (EFA), Long chain polyunsaturated fatty acids (LCPUFA), Docosahexaenoic acid (DHA), Eicosapentaenoic acid (EPA), Arachidonic acid (ARA), Linoleic acid (LA), Alpha Linolenic acid (ALA), Full term (FT) Preterm (PT), High socioeconomic status (HSE), Low socioeconomic status (LSE)

Competing Interests: The authors declare that there is no conflict of interest

Authors' Contributions: Shobha $\mathrm{S} \mathrm{Rao}^{1} \mathrm{PhD}$ is the principle investigator for this study and contributed to fundamental conceptualization of the study, provided oversight for statistical analysis and writing the manuscript. Prachi S Ranade MSc. Research Scholar, is the research coordinator for the study and conducted all field and laboratory work, performed statistical analysis and writing of manuscript. 
Acknowledgements and Funding: PSR was supported by a Research fellowship by University Grant Commission, India. We thank Dr. Girish Godbole, MD Gynecology and staff of Deenanath Mangeshkar Hospital, Pune, India for making available the hospital records of the participating mothers and providing maternal blood samples.

\section{REFERENCES:}

1. Martin JA, Kochanek KD, Strobino DM, Guyer B, MacDorman MF: Annual Summary of Vital Statistics 2003. Pediatrics 2005, 115:619-634.

2. Bhargava SK, Singh KK, Saxena BN: National task collaborative studies on identification of high risk families, mothers, outcome of their offsprings with particular reference to maternal nutrition, LBW, perinatal morbidity and mortality in certain urban and slum communities. ICMR publication; 1990.

3. Gupta PC, Subramoney S: Smokeless tobacco use, birth weight, and gestational age: population based, prospective cohort study of 1217 women in Mumbai, India. Brit Med J 2004, 328: 1538-1540.

4. Singh U, Singh N, Seth S: A prospective analysis of etiology and outcome of preterm labor. J Obstet Gynecol India 2007, 57: 48-52.

5. McPheeters ML, Miller WC, Hartmann KE, Savitz D A, Kaufman J S, Garrett JM, and Thorp JM : The epidemiology of threatened preterm labor: a prospective cohort study. Am J Obstet Gynecol 2005, 192:1325-1329

6. Meis PJ, Michielutte R, Peters TJ, Wells HB, Sands RE, Coles EC, and Johns KA: Factors associated with preterm birth in Cardiff, Wales. II. Indicated and spontaneous preterm birth. Am J Obstet Gynecol 1995, 173:597-602.

7. Hagan $\mathrm{R}$, Benninger $\mathrm{H}$, Chiffings $\mathrm{D}$, Evans $\mathrm{S}$, French N: Very preterm birth--a regional study. Part 1: Maternal and obstetric factors. Br J Obstet Gynaecol 1996, 103:230-238.

8. Honest H, Bachmann LM, Ngai C, Gupta JK, Kleijnen J, Khan KS: The accuracy of maternal anthropometry measurements as predictor for spontaneous preterm birth--a systematic review. Eur J Obstet Gynecol Reprod Biol 2005, 119:11-20.

9. Banhidy F, Acs N, Puho EH and Czeizel AE: Iron deficiency anemia: Pregnancy outcomes with or without iron supplementation. Nutrition 2011, 27: 65-72.

10. Morgan-Ortiz F, Cinco-Sanchez A, Douriet-Marin FA, Baez-Barraza J, MunozAcosta J, Osuna-Ramirez I: Sociodemographic and obstetric factors associated with preterm birth. Ginecol Obstet Mex 2010, 78:103-109.

11. Owens S, Fall CH: Consequences of poor maternal micronutrition before and during early pregnancy. Trans R Soc Trop Med Hyg 2008, 102:103-104.

12. Olsen SF, Hansen HS, Sorensen TI, Jensen B, Secher NJ, Sommer S, Knudsen LB: Intake of marine fat, rich in (n-3)-polyunsaturated fatty acids, may increase birthweight by prolonging gestation. Lancet 1986, 2:367-369.

13. Olsen SF, Hansen HS, Sommer S, Jensen B, Sorensen TI, Secher NJ, Zachariassen P: Gestational age in relation to marine $n-3$ fatty acids in maternal erythrocytes: a 
study of women in the Faroe Islands and Denmark. Am J Obstet Gynecol 1991, 164:1203-1209.

14. Olsen SF, Hansen HS, Secher NJ, Jensen B, Sandstrom B: Gestation length and birth weight in relation to intake of marine n-3 fatty acids. Br J Nutr 1995, 73:397-404.

15. Olsen SF, Osterdal ML, Salvig JD, Weber T, Tabor A, Secher NJ: Duration of pregnancy in relation to fish oil supplementation and habitual fish intake: a randomised clinical trial with fish oil. Eur J Clin Nutr 2007, 61:976-85.

16. Olsen SF, Secher NJ, Tabor A, Weber T, Walker JJ, Gluud C: Randomised clinical trials of fish oil supplementation in high risk pregnancies. Fish Oil Trials In Pregnancy (FOTIP) Team. Bjog 2000, 107:382-395.

17. Baguma-Nibasheka M, Brenna JT, Nathanielsz PW: Delay of preterm delivery in sheep by omega-3 long-chain polyunsaturates. Biol Reprod 1999, 60:698-701.

18. Helland IB, Saugstad OD, SmithL, Saarem K, Solvoll K, Ganes T, \& Drevon CA: Similar effects on infants of n-3 and n-6 fatty acids supplementation to pregnant and lactating women. Pediatrics. 2001, 108: E82.

19. Malcolm CA, McCulloch DA, Montgomery C, Shepherd A, Weaver LT: Maternal docosahexaenoic acid supplementation during pregnancy and visual evoked potential development in term infants: a double blind, prospective, randomized trial. Arch Dis Child Fetal Neonatal Ed. 2003, 88:383-90.

20. Rao S, Yajnik CS, Kanade A, Fall CH, Margetts BM, Jackson AA, Shier R, Joshi S, Rege S, Lubree H, and Desai B: Intake of micronutrient-rich foods in rural Indian mothers is associated with the size of their babies at birth: Pune Maternal Nutrition Study. J Nutr 2001, 131:1217-1224.

21. Indian Council of Medical Research: Ethical guidelines for biomedical research on human subjects. ICMR Publication, New Delhi; 2006, 1-7.

22. Folch J, Lees M, Sloane Stanley GH: A simple method for the isolation and purification of total lipides from animal tissues. J Biol Chem 1957, 226:497-509.

23. Association of Official Analytical Chemists: Official Methods of Analysis Section 26.052. 5. 10th edition. Washington, D.C; 1965.

24. Kanade AN, Rao S, Kelkar RS, Gupte S: Maternal nutrition and birth size among urban affluent and rural women in India. J Am Coll Nutr 2008, 27:137-45.

25. Nettleton JA: Omega-3 fatty acids and health: Chapman \& Hall; 1995.

26. Al MDM, van Houwelingen AC: Relation between birth order and the maternal and neonatal docosahexaenoic acid status Eur J Clin Nutr 1991, 51:548-553.

27. Berry C, Montgomery C, Sattar N, Norrie J, Weaver LT: Fatty acid status of women of reproductive age. Eur J Clin Nutr 2001, 55:518-524.

28. Ghafoorunissa: Fats in Indian diets and their nutritional and health implications. Lipids 1996, 31: S287-291.

29. Reece MS, McGregor JA, Allen KG, Harris MA: Maternal and perinatal long-chain fatty acids: possible roles in preterm birth. Am J Obstet Gynecol 1997, 176:907-914. 
30. Muthayya S, Dwarkanath P, Thomas T, Ramprakash S, Mehra R, Mhaskar A, Mhaskar R, Thomas A, Bhat S, Vaz M, \& Kurpad AV: The effect of fish and omega-3 LCPUFA intake on low birth weight in Indian pregnant women. Eur J Clin Nutr 2009, 63:340-346.

31. Olsen SF, Secher NJ: Low consumption of seafood in early pregnancy as a risk factor for preterm delivery: prospective cohort study. Brit Med J 2002, 324:447.

32. Araya J, Rojas M, Fernandez P, Mateluna A: Essential fatty acid content of maternal erythrocyte phospholipids: A study in preterm and full-term human newborns. Rev Med Chil 1998, 126:391-396.

33. Pontes PV, Torres AG, Trugo NM, Fonseca VM, Sichieri R: n-6 and n-3 Long-chain polyunsaturated fatty acids in the erythrocyte membrane of Brazilian preterm and term neonates and their mothers at delivery. Prostaglandins Leukot Essent Fatty Acids 2006, 74: 117-123.

34. Al-Tamer YY, Mahmood AA: Fatty-acid composition of the colostrum and serum of fullterm and preterm delivering Iraqi mothers. Eur J Clin Nutr 2004, 58:1119-1124.

35. Arab L: Biomarkers of fat and fatty acid intake. J Nutr 2003, 133:925S-932S. 